01,07

\title{
Ab initio моделирование энергии растворения и энергии связи водорода с 3sp-, 3d- и $4 d$-примесями в ОЦК-железе
}

\author{
(C) М.С. Ракитин ${ }^{1,2}$, А.А. Мирзоев ${ }^{1, \uparrow}$ \\ Южно-Уральский государственный университет, \\ Челябинск, Россия \\ ${ }^{2}$ Брукхейвенская Национальная Лаборатория, \\ Аптон, Нью-Йорк, США \\ ฯ E-mail: mirzoevaa@susu.ru
}

Поступила в Редакцию 18 февраля 2021 г.

В окончательной редакции 18 февраля 2021 г.

Принята к публикации 25 февраля 2021 г.

\begin{abstract}
Фундаментальное понимание локализации атомов Н в стали - важный шаг к теоретическому описанию механизмов водородного охрупчивания на атомном уровне. Мы исследуем с помощью ab initio расчетов в рамках теории функционала плотности (DFT) влияние различных примесей замещения $\mathrm{Mg}, \mathrm{Al}, \mathrm{Si}, \mathrm{Sc}, \mathrm{Ti}, \mathrm{V}$, $\mathrm{Cr}, \mathrm{Mn}, \mathrm{Co}, \mathrm{Ni}, \mathrm{Cu}, \mathrm{Zn}, \mathrm{Y}, \mathrm{Zr}, \mathrm{Nb}, \mathrm{Mo}, \mathrm{Pd}$ и $\mathrm{Cd}$ на энергию растворения водорода в решетке ОЦК-железа. Выделены электронный и упругий вклады различных примесей в энергию растворения, проанализировано их влияние на энергию связи водорода и примесей. Показано существование линейной зависимости энергии растворения водорода от величины изменения электронной плотности внутри тетраэдрической поры, после внедрения в нее атома водорода. Полученные результаты позволили сформулировать ключевые механизмы управления локализацией водорода в ОЦК-железе легирующими примесями замещения.
\end{abstract}

Ключевые слова: $a b$ initio моделирование, водород, ОЦК-железо, примеси замещения энергия связи, энергия растворения.

DOI: 10.21883/FTT.2021.07.51031.034

\section{1. Введение}

Проблема водорода и металлов и, в частности, явление, известное как „водородная хрупкость“ сталей [1-4], является одновременно очень старой и очень новой проблемой. Его природа [5-8] связана с выделением при кристаллизации вблизи дефектов решетки газообразного водорода, захваченного расплавом из атмосферы. Водородное охрупчивание интересует металлургов и физиков уже более ста лет, предложено несколько механизмов его возникновения, но построить законченную теорию явления так и не удалось. В последнее время новый виток интереса к указанной проблеме связан с расширением сети магистральных газопроводов, по которым перекачиваются водородсодержащие смеси, а в дальнейшем, при переходе к экологически чистой водородной энергетике, планируется перекачка смеси водород-метан [9].

Каким бы ни был механизм охрупчивания стали, в области зарождения трещины должна быть достигнута критическая концентрация водорода [4]. Кроме того, требуется, чтобы скорость переноса Н была достаточно высокой для поддержания этой критической концентрации. Однако объем и скорость переноса Н в кристаллическом материале управляется процессами его захвата точечными и протяженными дефектами кристалла, микропустотами или дисперсными выделениями $[4,10,11]$. Таким образом, захват водорода в ловушки, внедренные в объем металла, может использоваться в качестве важного метода борьбы с эффектом охрупчивания ста- лей. В связи с этим, знание энергии захвата водорода различными примесями, часто содержащимися в сталях, является одним из важных моментов в понимании явления охрупчивания и борьбы с ним.

В предшествующей работе [12] в рамках теории функционала плотности в пакете WIEN-2k нами было проведено компьютерное моделирование энергии захвата водорода примесями $3 s p$-, $3 d$ - и $4 d$-металлов в матрице ОЦК-железа. Было показано, что водород занимает только тетраэдрические поры решетки. При выделении электронного (химического) и упругого вклада в энергию растворения и захвата водорода примесями, было показано, что оба вклада сопоставимы по величине и могут конкурировать друг с другом. Полученные в [12] результаты, так же как и близкие к ним данные ab initio моделирования работы [13], представляют собой полезный объем важной информации, физического объяснения которой не было, однако, дано. В настоящей работе сделана попытка более глубокого анализа Н-захвата в железе различными примесями. Показано, что изменение энергии растворения водорода, вызываемое примесью, можно связать с создаваемым ею возмущением электронной плотности матрицы.

\section{2. Постановка задачи и детали вычислений}

На рис. 1 представлена зависимости энергии растворения водорода в ОЦК-железе, легированном различными 


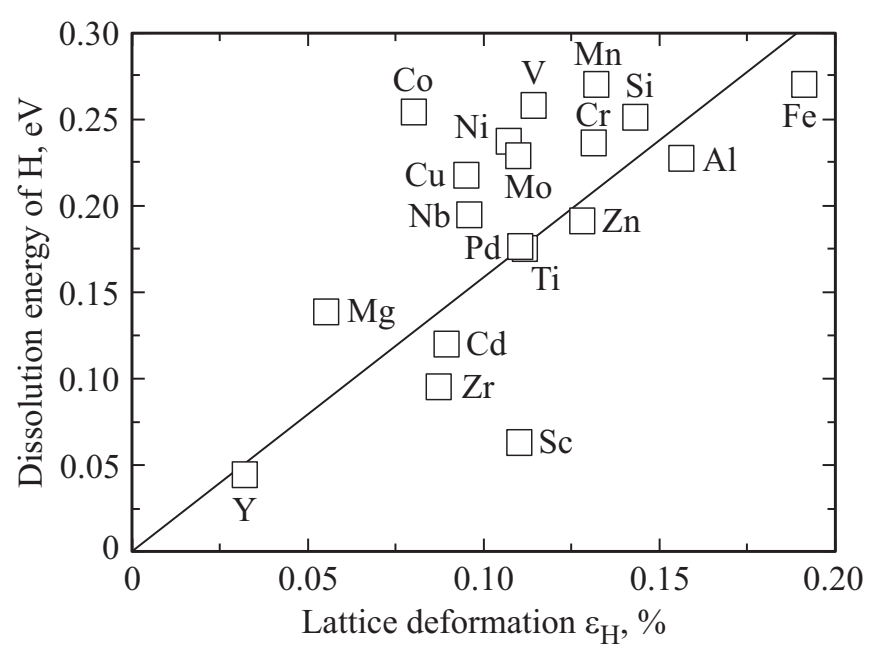

Рис. 1. Зависимость энергии растворения водорода в ОЦКсплавах железа, легированных примесями, названия которых указаны в квадратах, от относительной деформации решетки легированного сплава при растворении в нем водорода.

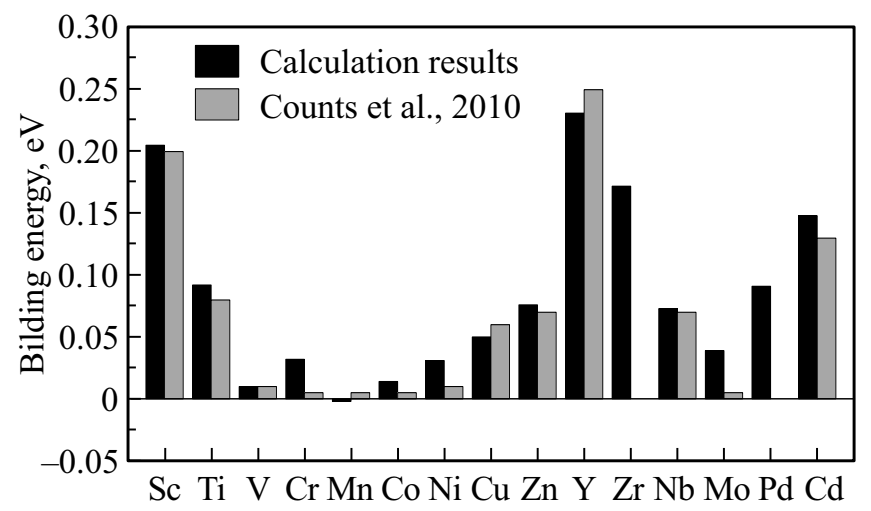

Рис. 2. Сравнение результатов моделирования энергии связи атомов водорода с примесями замещения в ОЦК-железе с данными работы [13].

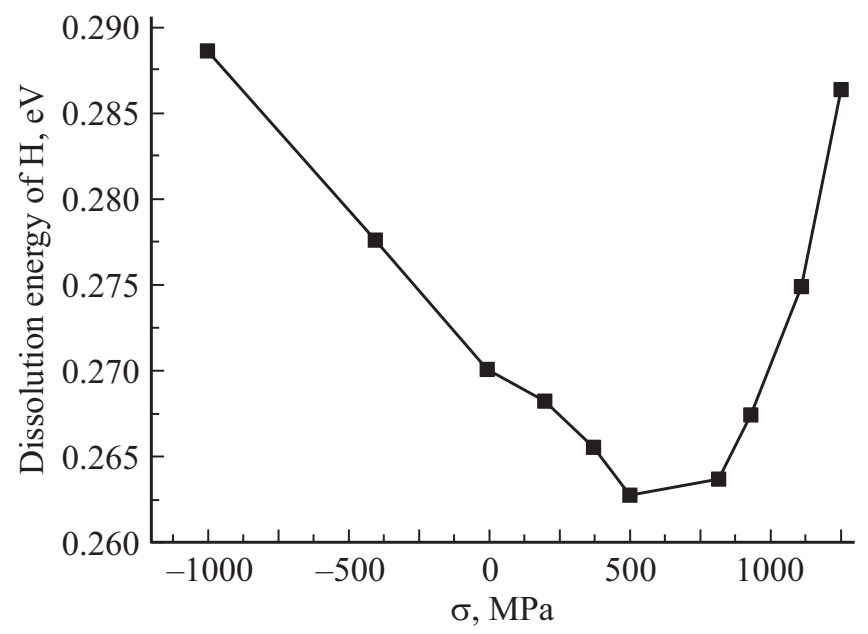

Рис. 3. Зависимость энергии растворения водорода в ОЦК-Fе от приложенного упругого напряжения. примесями, от величины деформации решетки, возникающей при помещении атома водорода в тетраэдрическую позицию вблизи примесного атома из работы [12]. Позиция выбиралась исходя из достижения наименьшей полной энергии системы. Видно, что возникающая при внедрении атома Н упругая энергия не является единственным фактором, определяющим величину энергии растворения водорода.

Об этом же свидетельствует и рис. 2, также взятый из [12], на котором представлены значения энергии захвата водорода всеми изученными примесями. Действительно, примесь Y, обладающая большим атомным радиусом, хорошо связывает водород, тогда как примеси $\mathrm{Mn}$ и Со с радиусом близким к атомному радиусу железа связь не образуют.

Энергия захвата, или энергия связи, водорода с примесью определялась формулой

$$
E_{\text {bind }}(\mathrm{H}-X)=E\left(\mathrm{Fe}_{54} \mathrm{H}\right)-E\left(\mathrm{Fe}_{53} X \mathrm{H}\right),
$$

$E_{\text {bind }}(\mathrm{H}-X)$ - энергия связи водорода с примесью $X$ в ОЦК-железе; $E\left(\mathrm{Fe}_{54} \mathrm{H}\right)$ - полная энергия суперьячейки из 54 атомов железа и внедренным в тетрапору атомом водорода, $E\left(\mathrm{Fe}_{53} X \mathrm{H}\right)$ - энергия той же суперъячейки, но с заменой одного атома железа на атом примеси $X$.

Чтобы прояснить ситуацию был проведен расчет энергии растворения водорода для разных параметров решетки чистого ОЦК-Fе. Возникающее при равномерной деформации решетки упругое напряжение находили как $\sigma=3 K$, где $K$ - объемный модуль упругости железа, для которого мы использовали экспериментальное значение $K=162 \mathrm{GPa}$. На основе этих отношений была построена представленная на рис. 3 зависимость энергии растворения водорода от напряжения, действующего на моделируемую суперьячейку. Приведенная зависимость состоит из двух четко выделенных частей. Нисходящий участок от -1000 до $+500 \mathrm{GPa}$ описывает хорошо известную линейную зависимость энергии растворения водорода от гидростатической деформации решетки [14]. Восходящий участок имеет совершенно иную природу и, скорее всего, связан с увеличением электронной плотности $n$ внутри тетраэдрической поры. В пользу этой гипотезы говорит успешное применение полуэмпирической модели эффективной среды [15], в которой энергия встраивания водорода в матрицу металла с плотностью электронного газа $n$ резко растет с увеличением этой величины.

Кроме того мы построили зависимость энергии захвата водорода от изменения объема решетки ОЦК-железа, при введении в нее легирующей примеси (рис. 4).

Наблюдается четкая, почти линейная корреляция между энергией взаимодействия водород-примесь и степенью расширения этой примесью решетки железа. В то же время, обнаруженные линейные зависимости имеют слегка различающийся наклон, и сдвинуты друг относительно друга для $3 d-, 4 d$-примесей и $3 s p$-элементов. Это означает, что кроме упругой энергии есть еще один фактор, который зависит от положения примеси 


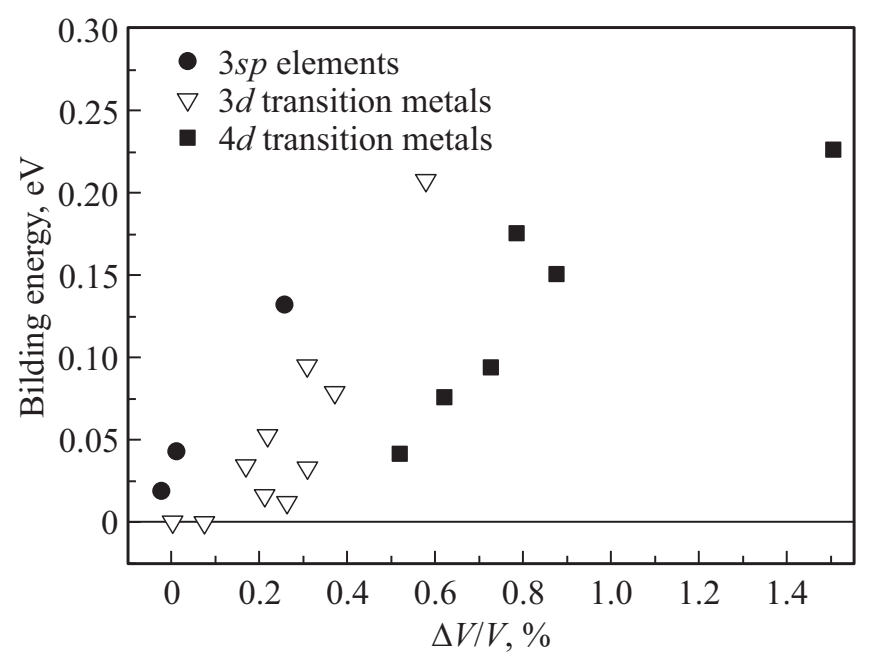

Рис. 4. Зависимость энергии связи атома водорода с легирующей примесью как функция величины относительной деформации решетки ОЦК-железа при растворении в ней данной примеси.

в Периодической системе, т. е. от числа валентных электронов примеси. Следовательно, вклад поля деформации решетки в зависимости от знака (растяжение или сжатие) может содержать как упругий, так и электронный вклады в энергию растворения водорода. Несмотря на то, что такая гипотеза была сформулирована довольно давно [16], изучение вкладов и сравнение их относительных значений до сих пор является сложной задачей.

Таким образом, совершенно ясно, что энергия растворения Н и энергия его связи с примесями зависит от локальной электронной плотности и энергии локальной деформации, причем последняя, в свою очередь, также может зависеть от электронной плотности.

Обнаруженные закономерности поставили перед нами следующую задачу: рассчитать электронную плотность в системе железо-примесь замещения и в системе железо-примесь-водород, чтобы оценить влияние электронной плотности на энергию захвата водорода примесью. С этой целью мы провели расчеты изменения плотности валентных электронов в объеме тетрапоры, после внедрения туда атома водорода.

$$
\Delta n=n_{\mathrm{Fe}_{53} X}-n_{\mathrm{Fe}_{54}}-n_{\mathrm{H}},
$$

где $n_{\mathrm{Fe}_{53} X}-$ плотность заряда валентных электронов в суперъячейке ОЦК-железа с одной из исследуемых примесей замещения $\mathrm{Fe}_{53} X ; n_{\mathrm{Fe}_{54}}-$ плотность заряда в суперьячейке чистого железа; $n_{\mathrm{H}}-$ плотность заряда в суперъячейке, содержащей одиночный атом водорода. Во всех случаях использовалась суперьячейка с постоянной геометрией, а плотность заряда вычислялась для объема тетрапоры ОЦК-решетки железа.

\section{3. Результаты расчетов}

Используя возможности пакета моделирования VASP $[17,18]$, мы провели расчеты изменения элект- ронной плотности валентных электронов, вызванной внедрением атома водорода, внутри тетраэдрических пор, расположенных в различных координационных сферах примесей замещения. Все расчеты проводились для ферромагнитной кубической ОЦК суперъячейки, содержащей 54 атома с применением PAW-потенциалов [19] и обобщенного градиентного приближения GGA-PBE [20] с отсечкой энергии плоских волн при $450 \mathrm{eV}$. Результаты расчета в соответствии с формулой (2) представлены на рис. 5.

Отметим, что во всех рассмотренных случаях изменение электронной плотности при добавлении водорода положительно. Это означает, что водород в ОЦК-железе является донором, частично передавая свою электронную плотность в коллективизированные состояния матрицы. Видно также, что все изученные примеси образуют общий линейный тренд, хотя и с некоторым разбросом, скорее всего, из-за неизбежных погрешностей расчетов. Этот результат является одним из основных в настоящем исследовании. Впервые с помощью $a b$ initio расчетов обнаружена универсальная зависимость: энергия растворения водорода тем меньше, чем меньше внедренный атом водорода меняет электронную плотность в тетраэдрической поре. По-видимому, именно наличие такой закономерности обеспечило в свое время успех методам эффективной среды.

Энергия связи атома водорода с примесью замещения для $3 d$-примесей равна разности энергий растворения водорода в чистом железе и в железе, содержащем примеси замещения. Поскольку первая величина одинакова для всех примесей, то все факторы, связанные с влиянием примесей на энергию растворения в ОЦК-Fе, которые мы обсуждали выше, влияют также и на энергию связи. Отсюда следует, что энергия связи будет тем больше,

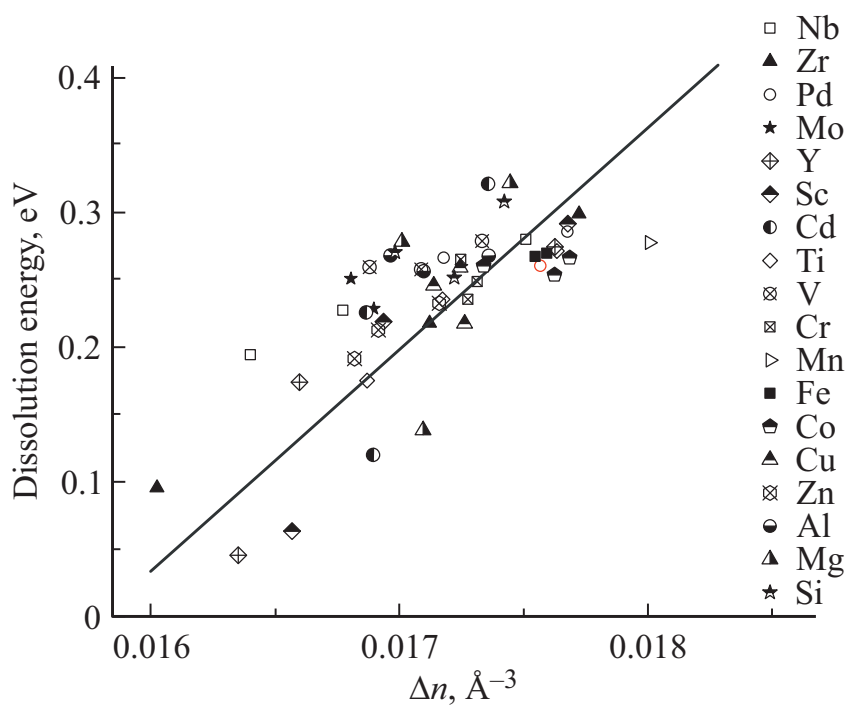

Рис. 5. Энергия растворения водорода вблизи различных примесей в матрице железа как функция изменения электронной плотности внутри соответствующей тетраэдрической поры, после внедрения в нее атома водорода. 
чем меньше будет возмущение электронной плотности при растворении Н в легированном сплаве в сравнении со значением $0.0175 \AA^{-3}$ для чистого железа. Конечно, таким свойством будут обладать, прежде всего, примеси с большим атомным радиусом, которые, локально расширяя решетку матрицы вокруг себя, снижают давление на внедренный атом Н, уменьшая тем самым перенос с него заряда и изменение электронной плотности. Однако, возможен и второй механизм, когда легирование приводит к сдвигу уровня Ферми системы в локальный минимум плотности состояний, что делает невыгодным перенос электронной плотности от Н к матрице. Именно этим, скорее всего, объясняется повышенное значение энергии связи при легировании примесями $\mathrm{Cu}$ и $\mathrm{Zn}$.

\section{4. Заключение}

C помощью $a b$ initio расчетов в рамках теории функционала плотности мы исследовали влияние малых концентраций примесей замещения $\mathrm{Mg}, \mathrm{Al}, \mathrm{Si}, \mathrm{Sc}, \mathrm{Ti}, \mathrm{V}$, $\mathrm{Cr}, \mathrm{Mn}, \mathrm{Co}, \mathrm{Ni}, \mathrm{Cu}, \mathrm{Zn}, \mathrm{Y}, \mathrm{Zr}, \mathrm{Nb}, \mathrm{Mo}, \mathrm{Pd}$ и $\mathrm{Cd}$ на энергию растворения водорода в ОЦК-железе и энергии захвата водорода указанными примесями. При этом впервые были определены энергии связи $\mathrm{H}-\mathrm{Pd}(0.087 \mathrm{eV})$ и связи $\mathrm{H}-\mathrm{Zr}(0.17 \mathrm{eV})$.

Показано, что наибольшая энергия захвата водорода соответствует примесям замещения $\mathrm{Y}, \mathrm{Sc}, \mathrm{Zr}, \mathrm{Cd}, \mathrm{Ti}$ и Mg. Проведенный в работе анализ показал, что энергия связи водорода с легирующими примесями в ОЦКжелезе будет тем больше, чем меньшее возмущение электронной плотности возникает при растворении Н в легированном сплаве. Отсюда следует, что поиск примесей, допускающих сильный захват водорода, следует вести в двух направлениях. В первую очередь следует рассматривать примеси, которые либо понижают плотность электронных состояний (DOS) сплава на уровне Ферми, либо, благодаря большому атомному радиусу, способны локально увеличивать вблизи себя объем тетрапор в решетке матрицы.

\section{Финансирование работы}

Работа поддержана грантом РФФИ № 20-43740004 p_a_Челябинск.

\section{Конфликт интересов}

Авторы заявляют, что у них нет конфликта интересов.

\section{Список литературы}

[1] J.P. Hirth. Met. 1. Trans. A 11, 861 (1980).

[2] A.R. Troiano. Trans. ASM 52, 54 (1960).

[3] H. Vehoff. In: Hydrogen in Metals III: Properties and Applications / Ed. H. Wipf. Springer, Berlin, Heidelberg. (1997). $215 \mathrm{p}$.
[4] Y. Fukai The Metal-Hydrogen System: Basic Bulk Properties. Springer-Verlag. Berlin Heidelberg. (2005). 202 p.

[5] H.K. Birnbaum, P. Sofronis. Mater. Sci. Eng. A 176, 191 (1994).

[6] S.P. Lynch. Met. Trans. 3, 189 (1979).

[7] I.M. Bernstein, A.W. Thompson. In: Hydrogen embrittlement and stress corrosion cracking / Ed. R. Gibala, R.F. Hehemann. Am. Soc. Met. Met. Park, OH. (1984). 135 p.

[8] W. Gerberich. In: Modeling hydrogen induced damage mechanisms in metals. Gaseous Hydrogen Embrittlement of Materials in Energy Technologies / Ed. R.P. Gangloff, B.P. Somerday. Woodhead Publishing Limited. 209 (2012). 209 p.

[9] N. Nanninga, A. Slifka, Y. Levy, C. White. J. Res. Nat. Institute Standards Technology 115, 6, 437 (2010).

[10] S.M. Myers, M.I. Baskes, H.K. Birnbaum, J.W.Corbett, G.G. DeLeo, S.K. Estreicher, E.E. Haller, P. Jena, N.M. Johnson, R. Kirchheim, S.J. Pearton, M.J. Stavola. Rev. Mod. Phys. 64, 559 (1992).

[11] D.A. Mirzaev, A.A. Mirzoev, K.Yu. Okishev, M.S. Rakitin. Mol. Phys. 110, 1299 (2012).

[12] Д.А. Мирзаев, А.А. Мирзоев, М.С. Ракитин. Вестн. ЮУрГУ. Сер. Металлургия 16, 4, 40 (2016).

[13] W.A. Counts, C. Wolverton, R. Gibala. Acta Materialia 58, 4730 (2010).

[14] D. Psiachos, T. Hammerschmidt, R.Drautz. Acta Materialia 59, 4255 (2011).

[15] J.K. Norskov, F. Besenbacher. J. Less Commun Met. 130, 475 (1987).

[16] P. Jena, R.M. Nieminen, M.J. Puska, M. Manninen. Phys. Rev. B. 3, 7612 (1985).

[17] G. Kresse, J. Furthmüller. Phys. Rev. B 54, 16, 11169 (1996).

[18] J. Hafner. J. Comput. Chem. 29, 13, 2044 (2008).

[19] P.E. Blöchl. Phys. Rev. B 50, 24, 17953 (1994).

[20] J.P. Perdew, K. Burke, M. Ernzerhof. Phys. Rev. Lett. 7, 3865 (1996).

Редактор Д.В. Жуманов 